\title{
Improved insulin sensitivity, central systolic pressure and inflammatory indicators achieved with minor weight reduction in overweight and obese subjects given education on lifestyle modification
}

\author{
Farah Diana Ariffin ${ }^{\mathrm{a}}$, AAA Ismail ${ }^{\mathrm{b}}$, Vina Tan Phei Sean ${ }^{\mathrm{c}}$, Zurkurnai Yusoff ${ }^{\mathrm{d}}$, Siti Azima Awang ${ }^{\mathrm{e}}$, Wan Rimei \\ Wan Abdul Ranie ${ }^{\text {, Aida Hanum Ghulam Rasool }}{ }^{\mathrm{a}}$ \\ ${ }^{a}$ Pharmacology Vascular Laboratory, ${ }^{b}$ Community Medicine Department, ${ }^{d}$ Department of Medicine, \\ School of Medical Sciences, 'School of Health Sciences, ${ }^{e}$ Dietetic Unit of Hospital Universiti Sains \\ Malaysia, Universiti Sains Malaysia, Health Campus, Kota Bharu 16150, Malaysia
}

\begin{abstract}
Background: Obesity is a global epidemic disease; lifestyle modification is an approach in the prevention and management of obesity.

Objective: We determined the effects of education on modified lifestyle intervention on arterial stiffness, metabolic and inflammatory markers.

Methods: Twenty-five generally healthy overweight and obese subjects completed nine months education on modified lifestyle intervention at Hospital Universiti Sains Malaysia, Kota Bharu. Subjects were regularly counselled to increase physical activity and modify their diet during intervention. Arterial stiffness was measured noninvasively using carotid femoral pulse wave velocity (PWV) and pulse wave analysis (PWA). Anthropometric measurements, body fat percentage and visceral fat, central and brachial blood pressures, lipid profile, the inflammatory marker high sensitivity C-reactive protein (hsCRP) and insulin sensitivity were also recorded.

Results: After nine months, a significant weight loss of $2.2 \mathrm{~kg}$ was observed associated with significant reductions in waist and hip circumference, aortic systolic blood pressure, serum fasting insulin, insulin resistance, and hsCRP levels. Insulin sensitivity was increased, while body fat and visceral fat percentages were marginally reduced $(p=0.058$ and $p=0.059)$. No significant differences were seen in arterial stiffness, fasting plasma glucose and lipid profile.

Conclusion: Education on modified lifestyle intervention improved insulin sensitivity and resistance, reduced hsCRP and aortic systolic blood pressure despite the small weight reduction achieved.
\end{abstract}

Keywords: Arterial stiffness, education on lifestyle modification, hsCRP, insulin sensitivity and resistance, obesity

Obesity is a risk factor for type II diabetes, coronary heart disease (CHD), hypertension, stroke, osteoarthritis, gallbladder disease, sleep apnea, and certain forms of cancer. Obesity is associated with increased arterial stiffness [1,2] and inflammation $[3,4]$ as well as altered metabolic profile [5], all of which predisposes to cardiovascular and metabolic complications. The World Health Organization(WHO) reported that at least 2.8 million people die each year as a result of being overweight or obese.

Correspondence to: Prof. Aida Hanum Ghulam Rasool, Pharmacology Department, School of Medical Sciences, Universiti Sains Malaysia, Kota Bharu 16150, Malaysia. E-mail: aidakb@usm.my
Lifestyle modification is one approach in prevention and management of obesity. Most previous reported studies on lifestyle modification were conducted with regimented dietary and exercise interventions for periods of less than six months [6-9]. In this study, we examined the effects of nine months education on lifestyle modification to reduce weight on waist circumference (WC), hip circumference (HC), body fat and visceral fat percentages, arterial stiffness, metabolic and inflammatory markers. Subjects were regularly counselled on good dietary habits and increasing physical activity for 9 months. Measurements of arterial stiffness, fasting plasma glucose (FPG), fasting insulin levels, insulin sensitivity and resistance, lipid 
profile and serum hsCRP were determined during the nine months intervention.

\section{Materials and methods Subjects}

Thirty-four (29 females and 5 males) generally healthy overweight and obese subjects based on an Asia-Pacific classification [10] participated in this study. Two were hypertensive; one was treated with amlodipine $10 \mathrm{mg}$ daily while the other was treated with atenolol $50 \mathrm{mg}$ daily. Other subjects were not taking regular medication. The study protocol was approved by the Human Ethical Committee of Universiti Sains Malaysia and all subjects provided written and signed informed consent. This study was performed at the School of Medical Sciences and Universiti Sains Malaysia Hospital (HUSM), Universiti Sains Malaysia Health Campus, Kota Bharu. Subjects were recruited from the Obesity Clinic of HUSM and nearby health clinics. All were between 18 to 60 years of age with systolic blood pressure (SBP) $<146 \mathrm{mmHg}$ and diastolic blood pressure (DBP) $<95 \mathrm{mmHg}$ with or without treatment. Subjects were excluded if they had diabetes or FPG $>6.1 \mathrm{mmol} / 1$, cardiovascular diseases or suffered from chronic medical conditions. Subjects who were on lipid lowering agents, angiotensin-converting enzyme (ACE) inhibitors, and nonsteroidal antiinflammatory drugs (NSAIDs) were excluded. Pregnant and women planning to be pregnant and patients who were taking antiobesity medications or who had stopped antiobesity medications within the last two months of the recruitment period were also excluded.

\section{Study protocol}

Before intervention, all subjects performed an exercise stress test using a modified Bruce protocol to assess their ability to exercise. Eligible subjects then attended a baseline study session where measurements for anthropometric profile, body and visceral fat percentages, arterial stiffness, brachial and central blood pressures, and blood samples for FPG, lipid profile, fasting insulin, and hsCRP were performed. Anthropometric measurements, body composition, arterial stiffness, brachial and central blood pressures were repeated at 3,6, and 9 months' of intervention while blood parameters were repeated at 6 and 9 months. Subjects were asked to fast overnight and refrain from drinking coffee and consuming high salted food prior to each study visit.

\section{Education on lifestyle modification intervention program}

Subjects in this study were counseled by a sports science lecturer at baseline and monthly for the first 3 months, then every 3 months for the rest of the remaining 6 months. During the first consultation, subjects were given exercise prescription consisting of aerobic exercises based on their past exercise experience and physical activity, fitness level, and current lifestyle. Generally, they were asked to exercise for 30-40 minutes per session for at least three times per week. They were also given recommendations on ways to increase physical activity such as taking the stairs, encouraged to increase walking and performing household chores. Individual exercise prescription was reviewed at the next consultation; whether to continue with the current prescription or modified where necessary. Subjects were asked to start their exercise at an intensity of $40 \%$ " $50 \%$ of maximum heart rate and gradually increasing the intensity. Paffenberger's Physical Activities Questionnaire (PPAQ) [11] was filled by subjects to monitor their adherence in exercising. Dietary consultations were provided by two study dieticians, at baseline and every month for the first 3 months of intervention, then every 3 months for the rest of the remaining 6 months. The typical diet in many of our subjects prior to intervention consisted of three main meals; two are often rice based meals with side dishes, and another meal consisting of either bread, noodles, or cakes. A number of them enjoy food high in calorie content such as dishes containing coconut milk and fried food. Some of them would also snack in between the main meals. During consultations, subjects' eating habits and estimated calorie intake were assessed using a food frequency questionnaire and a food diary. These were performed to estimate their food intake and monitor their eating habits so as to be able to counsel the subjects better. Subjects were educated on the proper choice of food to be taken, ways to prepare healthy and balanced meals, how to calculate their daily calorie intake, and given guides on calorie contents of common Malaysian dishes. These were aimed to achieve a low caloric, low fat and cholesterol diet for subjects.

\section{Assessment of arterial stiffness}

A vascular study was performed in a quiet room at the Pharmacology Vascular Laboratory at an ambient temperature between $22^{\circ} \mathrm{C}$ to $23^{\circ} \mathrm{C}$. Subjects 
rested supine for at least 15 minutes before study measurements were taken. Assessment of arterial stiffness was performed using the parameters carotid femoral pulse wave velocity (PWV) and central augmentation index (AI) obtained from pulse wave analysis (PWA). Carotid femoral PWV and AI were measured noninvasively using a SphygmoCor device (Atcor Medical, Australia). All measurements for arterial stiffness were taken by a single investigator throughout the study.

Carotid femoral PWV measures the velocity of the pulse wave travel along the carotid-femoral segment by calculating the time delay between the pulse pressure waves at the two sites. Measurement of PWV was recorded by placing a pressure sensitive tonometer on the carotid followed by the femoral pulsations, and comparing the time delay at both sites against a simultaneously measured QRS complex (from electrocardiography). A higher PWV indicates increased aortic stiffness. The repeatability of the PWV measurement assessed as intra- and interday coefficient of variations were $5.1 \%$ and $4.9 \%$ respectively.

Pulse wave analysis was used to assess systemic arterial stiffness [12]. Applanation tonometry using a noninvasive high fidelity sensor (SPT-304, Millar Instruments, USA) was used to record the radial artery pressure waveform. A validated transfer function [12] was used to derive the aortic pressure waveform from the radial waveform, enabling aortic pressures and certain central arterial indices to be measured including augmentation index, aortic systolic and diastolic blood pressures. Central augmentation index is the proportion of central arterial pressure that results from arterial wave reflection and is commonly used to measure arterial stiffness [12]; a higher AI value indicates increased stiffness and vice versa. The repeatability of the AI measurement assessed as intraand interday coefficient of variations in this study were $7.5 \%$ and $5.7 \%$ respectively. Averages of two readings were recorded for the measurements of AI and PWV.

\section{Anthropometric measurements}

Body weight and height were measured using an SECA 789 weighing scale (Hamburg, Germany). Weight and height were taken to the nearest $0.1 \mathrm{~kg}$ and $0.1 \mathrm{~cm}$. Body mass index (BMI) was calculated as weight in $\mathrm{kg}$ divided by square of height $\left(\mathrm{kg} / \mathrm{m}^{2}\right)$. $\mathrm{WC}$ and $\mathrm{HC}$ were measured based on WHO recommendations [13]. WC $(\mathrm{cm})$ was taken at midway between the inferior margin of the last rib and the crest of the ilium in horizontal plane while $\mathrm{HC}$ $(\mathrm{cm})$ was taken at the level of the greatest posterior protuberance of the gluteals [14]. A body composition monitor with a scale (Omron Karada Scan Model HBF 361, Japan) was used to estimate body fat and visceral fat percentages using the principle of bioelectric impedance analysis (BIA). Sitting blood pressure (BP) was taken from the right arm of subjects using a digital blood pressure monitor (Omron MX3 (HEM-741C-C1)) after at least 10 minutes of rest. Blood pressure measurement was repeated twice at two minute intervals; average reading was recorded.

\section{Biochemical analysis}

Blood samples for lipid profile and FPG were analyzed on the same day of blood collection at the Chemical Pathology Laboratory of HUSM. Serum total cholesterol (TC) was estimated by using a cholesterol oxidase-peroxidase (CHOD-PAP) method. Serum triglycerides ( $\mathrm{Tg}$ ) were determined using a glycerol oxidase-peroxidase (GPO-PAP) method while HDL-cholesterol (HDL-C) was estimated by precipitant with a phosphotungstic reagent. The level of serum LDL-cholesterol (LDLC) was calculated using the Friedewald formula [15]. FPG was estimated using a glucose oxidase (GODPAD) method.

Serum for insulin and hsCRP were centrifuged (Hettich Universal 32 Tabletop Centrifuge, UK) at $4000 \mathrm{rpm}$ for 15 minutes and stored at $-80^{\circ} \mathrm{C}$ until assayed. Quantitative determination of serum hsCRP concentration was measured in duplicate using an enzyme-linked immunosorbent assay (ELISA) method (DRG CRP, HS C-Reactive Protein, USA). Serum fasting insulin was measured using a solid-phase, enzyme-labeled chemiluminescent immunometric assay on an Immulite 1000 Immunoassay System (Siemens Healthcare Diagnostics, USA). Homeostasis model assessment (HOMA) software was used to calculate insulin sensitivity (HOMA $\%$ S) using fasting insulin $(\mathrm{pmol} / \mathrm{L})$ and FPG $(\mathrm{mmol} / \mathrm{L})$ values [16]. HOMA for insulin resistance (HOMA-IR) was calculated manually using the following formula (16): HOMA-IR = fasting plasma glucose $(\mathrm{mmol} / \mathrm{L}) \times$ fasting insulin $(\mu \mathrm{IU} / \mathrm{ml}) \quad 22.5$.

\section{Statistical analysis}

Statistical analysis was performed using the Statistical Package for the Social Sciences software 
version 18.0 package for Windows (SPSS Inc, 2009). A repeated measures analysis of variance (ANOVA) and Friedman test were used to detect overall differences in parameter over the study time course of nine months. Results are expressed as mean (standard deviation) for normally distributed data and mean (interquartile range) for nonnormally distributed data. A $p<0.05$ was considered statistically significant.

\section{Results}

Of the 34 subjects who participated in the study, 25 completed the 9 months lifestyle intervention study. Nine subjects dropped out because of various reasons including pregnancy, noncompliance with the study protocol, or movement to a distant geographical location. Subjects who completed the study comprised of 4 males and 21 females with mean age of 36.8 (9.8) years and BMI values ranging from of $25.7 \mathrm{~kg} / \mathrm{m}^{2}$ to $43.7 \mathrm{~kg} / \mathrm{m}^{2}$.

Mean weight loss during 9 months intervention was $2.2 \mathrm{~kg}$ compared with baseline body weight, indicating a decrease of $3.3 \%$ of BMI unit. WC decreased significantly by $3 \%$ during intervention; a post hoc Bonferroni correction showed significant reduction occurred from baseline to 3 months $(p=0.039)$. Significant reduction in $\mathrm{HC}$ was seen with intervention; significant reductions were seen between baseline and 3 months $(p=0.002)$, between baseline and 6 months of intervention $(p=0.022)$ and between baseline and 9 months intervention $(p=0.029)$. Body fat percentage $(p=0.058)$ and visceral fat level $(p=0.059)$ were marginally decreased after 9 months intervention (Table 1).

Aortic systolic blood pressure (SAP) significantly decreased by $5 \mathrm{mmHg}$ after 9 months intervention with significant improvement occurring between 6 and 9 months of intervention $(p=0.029)$. Although not statistically significant, there was a trend of reduced peripheral SBP and DBP, and DAP during the 9 months intervention (Table 1). There were no significant changes in arterial stiffness parameters during the 9 months intervention.

The inflammatory marker, hsCRP was reduced significantly with intervention, with significant change occurring between baseline to 9 months $(p=0.020)$ (Table 2). Significant reductions were observed in fasting insulin level $(p=0.001)$ (Figure 1) and insulin resistance ( $p=0.007)$ (Figure 2$)$, while insulin sensitivity was significantly increased with intervention $(p=0.003)$ (Figure 3). TC, Tg, LDL-C, HDL-C, and FPG did not show any significant changes with intervention (Table 2).

Table 1. Clinical and vascular characteristics of subjects at baseline, 3, 6, and 9 months of intervention

\begin{tabular}{llllll}
\hline Parameters & Baseline & 3 months & 6 months & 9 months & Pvalue \\
\hline Weight, $\mathrm{kg}$ & $75.8(15.1)$ & $74.1(15.1)$ & $74.3(15.9)$ & $73.6(17.1)$ & $0.019^{*}$ \\
BMI, kg/m & $30.6(4.3)$ & $29.8(4.3)$ & $29.9(5.2)$ & $29.6(5.2)$ & $0.022^{*}$ \\
Waist, cm & $89.2(8.3)$ & $87.4(7.4)$ & $87.3(8.5)$ & $86.4(9.4)$ & $0.013^{*}$ \\
Hip, cm & $107.2(10.0)$ & $104.8(10.1)$ & $105.4(10.6)$ & $104.0(11.8)$ & $0.005^{*}$ \\
BF, \% & $35.8(5.1)$ & $35.2(5.6)$ & $35.4(5.5)$ & $34.6(5.9)$ & 0.058 \\
VF, level & $12.8(5.3)$ & $12.2(5.3)$ & $12.5(5.9)$ & $12.2(6.3)$ & 0.059 \\
SBP, $m m H g$ & $120(13)$ & $116(13)$ & $116(11)$ & $114(12)$ & 0.132 \\
DBP, $\mathrm{mmHg}$ & $73(10)$ & $73(9)$ & $73(8)$ & $70(7)$ & 0.062 \\
SAP, $\mathrm{mmHg}$ & $110(14)$ & $107(14)$ & $108(12)$ & $105(13)$ & $0.020^{*}$ \\
DAP, $\mathrm{mmHg}$ & $74(10)$ & $74(9)$ & $74(8)$ & $71(7)$ & 0.071 \\
AI, \% & $17.4(18.7)$ & $18.9(17.9)$ & $19.4(18.0)$ & $18.9(14.0)$ & 0.248 \\
PWV, $\mathrm{ms}^{-1}$ & $8.9(1.5)$ & $8.7(1.4)$ & $9.1(1.7)$ & $8.9(1.6)$ & 0.816
\end{tabular}

Data are presented as mean (SD). BMI indicates body mass index; BF, body fat; VF, visceral fat; SBP, systolic blood pressure; DBP, diastolic blood pressure; SAP, systolic aortic pressure; DAP, diastolic aortic pressure; AI, augmentation index; PWV, pulse wave velocity. * Overall significant reduction during nine months intervention.

Significant multiple comparison adjustment using Bonferroni correction. 
Table 2. Lipid profile, FPG and hsCRP at baseline, 6, and 9 months of intervention

\begin{tabular}{lllll}
\hline Parameters & Baseline & 6 months & 9 months & Pvalue \\
\hline TC, mmol/l & $5.65(1.13)$ & $5.75(0.92)$ & $5.71(1.02)$ & 0.773 \\
Tg, mmol/l & $1.59(1.44)$ & $1.56(1.32)$ & $1.45(1.19)$ & 0.272 \\
LDL-C, mmol/l & $3.53(0.98)$ & $3.59(0.88)$ & $3.61(0.95)$ & 0.788 \\
HDL-C, mmol/l & $1.40(0.40)$ & $1.47(0.44)$ & $1.45(0.39)$ & 0.559 \\
FPG, mmol/l & $4.61(0.65)$ & $4.78(0.90)$ & $4.78(0.90)$ & 0.445 \\
hsCRP, mg/l & $9.41(12.1)$ & $4.25(6.33)$ & $7.63(9.87)$ & $0.010^{*}$ \\
\hline
\end{tabular}

Data are presented as mean (SD). TC indicates total cholesterol; Tg, triglycerides; LDL-C, low-density lipoprotein cholesterol; HDL-C, high-density lipoprotein cholesterol; FPG, fasting plasma glucose; hsCRP, high sensitivity $\mathrm{C}$-reactive protein. ${ }^{*}$ Overall significant reduction during nine months intervention. Significant multiple comparisons adjustment using Bonferroni correction.

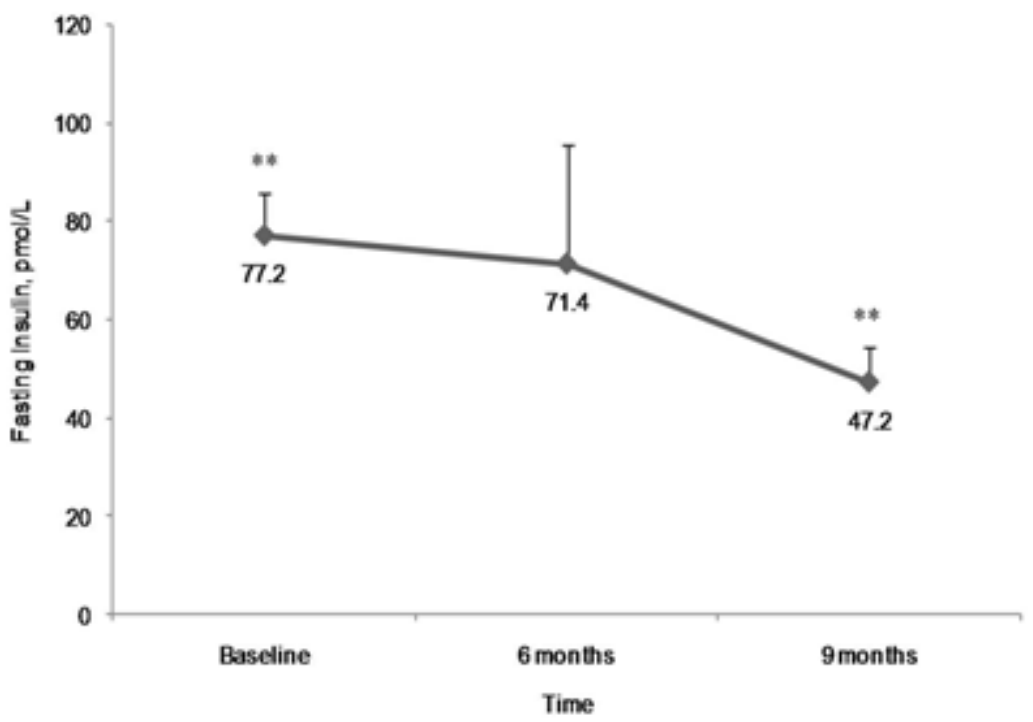

Figure 1. Mean fasting insulin levels at baseline, 6, and 9 months intervention. Significant reduction in insulin levels were seen after nine months intervention $(p=0.001)$.**Significant reduction between baseline and nine months using post hoc Bonferroni correction $(p<0.001)$. Error bars represent the standard errors of the means.

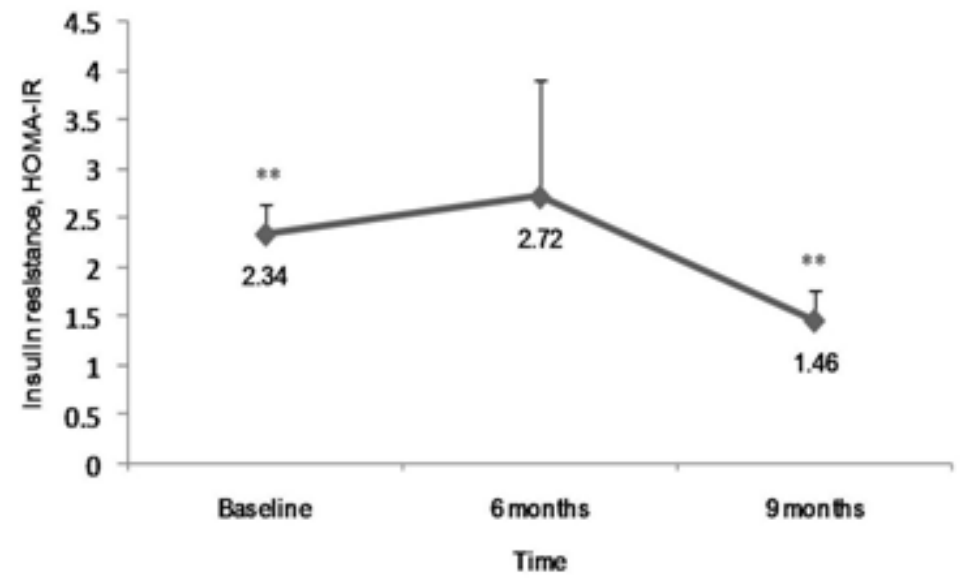

Figure 2. Mean insulin resistance (HOMA-IR) at baseline, 6 , and 9 months intervention. Significant reduction in insulin resistance was seen after nine months intervention $(p=0.007)$. * Significant reduction between baseline and 9 months $(p=0.006)$ using post hoc Bonferroni correction. Error bars represent the standard errors of the means. 


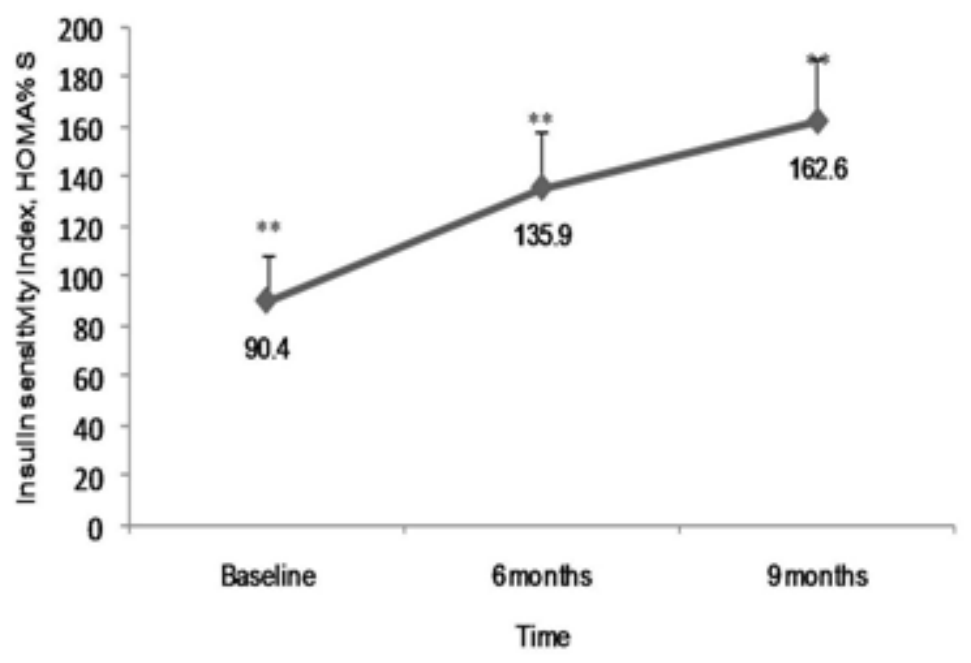

Figure 3. Mean insulin sensitivity index (HOMA\% S) at baseline, 6, and 9 months. Significant change in the insulin sensitivity index was seen after nine months intervention $(p=0.003)$.**Significant increments between baseline and six months $(p=0.007)$ and between baseline and nine months $(p<0.001)$ using post hoc Bonferroni correction. Error bars represent the standard errors of the means.

\section{Discussion}

Our results showed that body weight, BMI, WC, and $\mathrm{HC}$ were all significantly reduced after 9 months education on lifestyle modification. Body fat percentage and visceral fat levels were marginally reduced ( $p=0.058$ and $p=0.059$ respectively). Fasting insulin, insulin sensitivity, and resistance were all significantly improved during the 9 months intervention. Inflammatory markers measured as hsCRP and SAP were significantly reduced, while there were trends of improvement in DBP and DAP during the 9 months intervention. These positive changes occurred despite the relatively small weight loss seen at about 3\% from baseline, which was less than the perceived $5 \%$ figure deemed necessary to produce medical benefits with weight loss $[17,18]$. No improvements were seen in lipid profile parameters (TC, Tg, LDL-C, and HDL-C), FPG and arterial stiffness parameters during the 9 months intervention. These study findings showed that education on lifestyle modification could improve certain cardiovascular, metabolic, and inflammatory markers in overweight and obese subjects, despite the small weight reduction achieved.

The small weight reduction achieved in our study may be the result of our method of intervention that mainly educates, encourages, and helps subjects to modify and practice a healthy lifestyle for the long term by incorporating healthy practices in their own environment and daily activities. Studies of lifestyle modification with regimented and closely monitored dietary components and supervised physical activity appeared to produce more weight loss ranging from $7 \%$ to $9 \%$ from baseline compared with programs that were less structured, or self-monitored with weight loss ranging from $3 \%$ to $8 \%$ of baseline $[6,8$, 19-21]. Goldberg et al. [6] reported a moderate weight loss of about $9 \%$ in a 6 months supervised nutritional and exercise training sessions at a fitness centre. Dvorakova-Lorenzova et al. [19] showed that dietary education and supervised physical activity significantly decreased body weight by $7.7 \%$ of baseline body weight. In our study, subjects incorporated their dietary and physical activity programs into their daily lives based on recommendations and guidance given by dieticians and the sports science expert. We assume that many of our subjects lack adherence and discipline in changing and adapting to healthy dietary and increasing physical activity in their daily lives although they had been given adequate education, motivation, and assistance on these aspects. Easy access during the day and night to affordable food from the many food outlets available in Malaysia probably makes it difficult for subjects to maintain healthy dietary habits. Being a multiracial country, Malaysia has many festivities and feasts. These are likely to affect the recommended dietary regime in some of our subjects. Thus, overweight and obese subjects probably need better supervision with regimented programs in dietary and supervised physical activity to lose significant weight. 
Metabolic risk parameters, which were insulin sensitivity and resistance measured in this study both significantly improved during the 9 months intervention. Several studies reported weight loss ranging from $7 \%$ to $10 \%$ were able to improve insulin sensitivity and resistance [7, 22-24]. In the current study, fasting insulin level, insulin sensitivity and resistance were improved after 9 months intervention despite a small weight loss of $2.9 \%$ with no significant changes seen in FPG and lipid profile. Consistent with our study, Vogeser et al. [23] had reported reduced insulin level and resistance without improvement in FPG with a 9.8\% weight loss. Dengel et al. [22] reported that $7.8 \%$ weight loss significantly improved insulin sensitivity, but had no effect on FPG and insulin levels. Improvements in fasting insulin, insulin sensitivity, and resistance may be modulated by the reduction in abdominal fat, as shown by reductions in WC, body fat and visceral fat levels seen in our study. This was supported by Janssen et al. [25] who showed that visceral fat was positively associated with an oral glucose tolerance test (OGTT) glucose area, fasting insulin, and OGTT insulin area. Visceral fat level was negatively correlated with insulin sensitivity index. Adipose tissue contains 11 $\beta$-hydroxysteroid dehydrogenase type 1 (11ß-HSD1), which converts the inactive steroid metabolite, cortisone to the active steroid, cortisol. Elevated visceral fat increases endogenous $11 \beta$-HSD1, which increased cortisol levels. Elevated cortisol level contributes to the development of type 2 diabetes mellitus and insulin resistance [26], primarily by opposing the antigluconeogenic effects of insulin in the liver [27]. The $11 \beta$-HSD1 regeneration of glucocorticoids in visceral fat contributes substantially to the concentration of cortisol in the portal vein and is an important determinant of the development of insulin resistance associated with abdominal obesity [28].

The inflammatory marker, hsCRP was significantly reduced after intervention in this study. Reduction of hsCRP with weight reduction has been reported in previous studies [19, 29, 30]. We hypothesized that the improvement in hsCRP was modulated by a reduction in abdominal fat. In our study, WC was significantly reduced, while body fat percentage and visceral fat were marginally improved. Inflammation is independently linked with abdominal, especially visceral fat, possibly because of the regional variation in adipose tissue cytokine production [31]. Tchernof et al. [30] showed intraabdominal adipose tissue area in obese women was positively associated with CRP levels. Adipose tissue is an important source of circulating proinflammatory cytokines, such as interleukin (IL)-1, IL-6, tumor necrosis factor- $\alpha$, and other acute phase reactants, such as C-reactive protein. Secretion of IL-6 in adipose tissue has been proposed to be the link between plasma CRP and adiposity, as CRP synthesis in the liver is largely under the control of IL-6 [32]. Reduction of abdominal fat reduced the secretion of IL- 6 and thus reduced the CRP levels with weight loss. Chou et al. [33] and Yudkin et al. [34] showed that insulin resistance is strongly associated with CRP levels in nondiabetic subjects. In our study, reduction in hsCRP was associated with reduced fasting insulin and insulin resistance.

Aortic systolic BP significantly decreased in this study, with a trend of reduced peripheral DBP and aortic diastolic BP. Central systolic pressure had been shown to be a stronger predictor of cardiovascular structural damage and clinical outcomes compared to brachial BP in American Indians and European populations $[35,36]$. The small weight reduction achieved in our study may not be adequate to improve arterial stiffness parameters, AI and PWV. From prior studies, it appears that weight loss of $>7 \%$ may improve arterial stiffness and BP. This amount of weight loss was also associated with improvement in lipid profile, inflammatory markers, and insulin sensitivity $[7,22,37]$ achieved by a regimented nutritional and exercise intervention program [6]. Despite the improvement in hsCRP and insulin parameters, it is possible that the small weight loss achieved in this study and lack of improvement in lipid profile were not sufficient to improve arterial stiffness in our study. It is also possible that, at the relatively young mean age of 36.8 years in our study subjects, their arteries were not yet very stiff. Most were also relatively healthy, thus a small weight reduction without changes to the lipid profile may not significantly improve arterial stiffness in relatively healthy vessels.

Although our study showed improvements in inflammatory marker and insulin sensitivity, no improvement was seen in TC, Tg, LDL-C, or HDLC. These findings were supported by those of Fox [38] and Kaplan [39] who also reported no significant reduction in TC, Tg, or LDL-C with weight loss. This could be the result of the average baseline levels for TC, Tg, LDL-C, HDL-C, and FPG were already within normal limits, thus the small weight loss achieved 
did not result in further significant changes. Also, improvement in lipid profile appears not to only depend on weight reduction per se, but is likely to be dependent on the energy content and macronutrient distribution in subjects dietary intake [40].

We acknowledge several limitations to our study. Firstly, although Malaysia is a multiracial country consisting of three major races, namely Malays, Chinese, and Indians, all subjects who participated in this study were Malays. Prevalence of overweight and obesity in Malaysian was reported to be between $33.6 \%$ and $19.5 \%$, with the Indian and Malay ethnic groups having the highest prevalence for obesity at $24.6 \%$ and $23.2 \%$ respectively [41]. Our study was conducted in Kelantan, a state where approximately $95 \%$ of the population is Malay. Thus, whether similar responses to this intervention will occur among other races in Malaysia cannot be confirmed by this study. Secondly, out of the 25 subjects who had completed the study, 21 were female and only 4 were male. This probably reflects the gender trend of obese patients attending the Obesity Clinic of our hospital; where women are keener to seek assistance to lose weight compared with men. Another limitation of the current study is the lack of a control group. As those coming to the Obesity Clinic of HUSM seek assistance to reduce weight, it appears unethical to have a group of controls and deprive them of education, support and facilities needed to lose weight and improve their health.

\section{Conclusion}

Nine months education on modified lifestyle intervention reduced body weight, BMI, waist and hip circumference, and aortic systolic blood pressure. The small average weight loss of $2.2 \mathrm{~kg}$ was associated with reduced fasting insulin and resistance, increased insulin sensitivity, and reduction of the inflammatory marker, hsCRP. These findings can be used to educate people about the benefits of dietary modification and increasing physical activity that not only reduced body weight, but also had significant impact in reducing metabolic risk and probably obesity related cardiovascular comorbidities.

\section{Acknowledgment}

We thank Universiti Sains Malaysia for funding this study via the USM Research University Grant (1001/PPSP/812002), and Malaysian Ministry of Science, Technology and Innovation for providing the
National Science Fellowship to Farah Diana Ariffin.

\section{References}

1. Wildman RP, Mackey RH, Bostom A, Thompson T, Sutton-Tyrrell K. Measures of obesity are associated with vascular stiffness in young and older adults. Hypertension. 2003; 42:468-73.

2. Seifalian AM, Filippatos TD, Joshi J, Mikhailidis DP. Obesity and arterial compliance alterations. Curr Vasc Pharmacol. 2010; 8:155-68.

3. Al-Tahami BA, Bee YT, Ismail AA, Rasool AH. Impaired microvascular endothelial function in relatively young obese humans is associated with altered metabolic and inflammatory markers. Clin Hemorheol Microcirc. 2011; 47:87-97.

4. Ouchi N, Kihara S, Funahashi T, Matsuzawa Y, Walsh K. Obesity, adiponectin and vascular inflammatory disease. Curr Opin Lipidol. 2003; 14: 561-6.

5. Kahn SE, Prigeon RL, Schwartz RS, Fujimoto WY, Knopp RH, Brunzell JD, et al. Obesity, body fat distribution, insulin sensitivity and islet $\beta$-cell function as explanations for metabolic diversity. $\mathrm{J}$ Nutr. 2001; 131:354S-60S.

6. Goldberg Y, Boaz M, Matas Z, Goldberg I, Shargorodsky M. Weight loss induced by nutritional and exercise intervention decreases arterial stiffness in obese subjects. Clin Nutr. 2009; 28:21-5.

7. Barinas-Mitchell E, Kuller LH, Sutton-Tyrrell K, Hegazi R, Harper P, Mancino J, et al. Effect of weight loss and nutritional intervention on arterial stiffness in type 2 diabetes. Diabetes Care. 2006; 29:2218-22.

8. De Luis DA, Aller R, Izaola O, Gonzalez Sagrado M, Conde R, Perez Castrillon JL. Effects of lifestyle modification on adipocytokine levels in obese patients. Eur Rev Med Pharmacol Sci. 2008;12:33-9.

9. Lang HF, Chou CY, Sheu WH, Lin JY. Weight loss increased serum adiponectin but decreased lipid levels in obese subjects whose body mass index was lower than $30 \mathrm{~kg} / \mathrm{m}^{2}$. Nutr Res. 2011;31:378-86.

10. WHO/IASO/IOTF. The Asia-Pacific perspective: Redefining obesity and its treatment. Hong Kong: World Health Organization, International Obesity Task Force, International Association for the Study of Obesity; 2000.

11. Montoye HJ, Kemper HCG, Saris WH, Washburn RA. Measuring physical activity and energy expenditure: Human Kinetics Publishers; 1996.

12. O'Rourke MF, Gallagher DE. Pulse wave analysis. J Hypertens Suppl. 1996; 14:S147-57. Epub 1996/12/01. 
13. WHO. Physical status: the use and interpretation of anthropometry. Report of a WHO Expert Committee. Technical report series No. 854. Geneva: World Health Organization, 1995.

14. Norton K, Whittingham N, Carter L, Kerr D, Gore C, Marfell-Jones M. Measurement techniques in anthropometry. In: Norton K, Olds T, editors. Anthropometrica: a textbook of body measurement for sports and health courses. Sydney: UNSW Press; 1996. p. 27-73.

15. Friedewald WT, Levy RI, Fredrickson DS. Estimation of the concentration of low-density lipoprotein cholesterol in plasma, without use of the preparative ultracentrifuge. Clin Chem. 1972; 18:499-502.

16. Matthews DR, Hosker JP, Rudenski AS, Naylor BA, Treacher DF, Turner RC. Homeostasis model assessment: insulin resistance and $\beta$-cell function from fasting plasma glucose and insulin concentrations in man. Diabetologia. 1985; 28:412-9.

17. Wing RR, Lang W, Wadden TA, Safford M, Knowler WC, Bertoni AG, et al. Benefits of modest weight loss in improving cardiovascular risk factors in overweight and obese individuals with Type 2 diabetes. Diabetes Care. 2011; 34:1481-6.

18. Blackburn G. Effect of degree of weight loss on health benefits. Obes Res. 1995; 3 Suppl 2:211s-6s.

19. Dvorakova-Lorenzova A, Suchanek P, Havel PJ, Stavek P, Karasova L, Valenta Z, et al. The decrease in $\mathrm{C}$-reactive protein concentration after diet and physical activity induced weight reduction is associated with changes in plasma lipids, but not interleukin-6 or adiponectin. Metabolism. 2006; 55: 359-65.

20. Villareal DT, Miller BV, 3rd, Banks M, Fontana L, Sinacore DR, Klein S. Effect of lifestyle intervention on metabolic coronary heart disease risk factors in obese older adults. Am J Clin Nutr. 2006; 84:1317-23.

21. Andersen RE, Wadden TA, Bartlett SJ, Zemel B, Verde TJ, Franckowiak SC. Effects of lifestyle activity vs structured aerobic exercise in obese women: a randomized trial. JAMA. 1999; 281:335-40.

22. Dengel DR, Kelly AS, Olson TP, Kaiser DR, Dengel JL, Bank AJ. Effects of weight loss on insulin sensitivity and arterial stiffness in overweight adults. Metabolism. 2006; 55:907-11.

23. Vogeser M, Konig D, Frey I, Predel HG, Parhofer KG, Berg A. Fasting serum insulin and the homeostasis model of insulin resistance (HOMA-IR) in the monitoring of lifestyle interventions in obese persons. Clin Biochem. 2007; 40:964-8.
24. Utzschneider KM, Carr DB, Barsness SM, Kahn SE, Schwartz RS. Diet-induced weight loss is associated with an improvement in $\beta$-cell function in older men. J Clin Endocrinol Metab. 2004; 89:2704-10.

25. Janssen I, Fortier A, Hudson R, Ross R. Effects of an energy-restrictive diet with or without exercise on abdominal fat, intermuscular fat, and metabolic risk factors in obese women. Diabetes Care. 2002; 25:431-8.

26. Seckl JR, Morton NM, Chapman KE, Walker BR. Glucocorticoids and 11beta-hydroxysteroid dehydrogenase in adipose tissue. Recent Prog Horm Res. 2004; 59:359-93.

27. Qatanani M, Lazar MA. Mechanisms of obesityassociated insulin resistance: many choices on the menu. Genes Dev. 2007; 21:1443-55.

28. Wamil M, Seckl JR. Inhibition of $11 \beta$-hydroxysteroid dehydrogenase type 1 as a promising therapeutic target. Drug Discov Today. 2007; 12:504-20.

29. Jae SY, Fernhall B, Heffernan KS, Jeong M, Chun EM, Sung J, et al. Effects of lifestyle modifications on C-reactive protein: contribution of weight loss and improved aerobic capacity. Metabolism. 2006; 55: 825-31.

30. Tchernof A, Nolan A, Sites CK, Ades PA, Poehlman ET. Weight loss reduces C-reactive protein levels in obese postmenopausal women. Circulation. 2002; 105 : 564-9.

31. You T, Nicklas BJ. Chronic inflammation: role of adipose tissue and modulation by weight loss. Curr Diabetes Rev. 2006;2:29-37.

32. Yudkin JS, Kumari M, Humphries SE, Mohamed-Ali V. Inflammation, obesity, stress and coronary heart disease: is interleukin-6 the link? Atherosclerosis. 2000; 148:209-14.

33. Chou HH, Hsu LA, Liu CJ, Teng MS, Wu S, Ko YL. Insulin resistance is associated with $\mathrm{C}$-reactive protein independent of abdominal obesity in nondiabetic Taiwanese. Metabolism. 2009; 59:824-30.

34. Yudkin JS, Stehouwer CD, Emeis JJ, Coppack SW. C-reactive protein in healthy subjects: associations with obesity, insulin resistance, and endothelial dysfunction: a potential role for cytokines originating from adipose tissue Arterioscler Thromb Vasc Biol. 1999; 19:972-8.

35. Roman MJ, Devereux RB, Kizer JR, Lee ET, Galloway JM, Ali T, et al. Central pressure more strongly relates to vascular disease and outcome than does brachial pressure: the Strong Heart Study. Hypertension. 2007; 50:197-203.

36. Safar ME, Blacher J, Pannier B, Guerin AP, Marchais 
SJ, Guyonvarc'h PM, et al. Central pulse pressure and mortality in end-stage renal disease. Hypertension. 2002; 39:735-8.

37. Shargorodsky M, Fleed A, Boaz M, Gavish D, Zimlichman R. The effect of a rapid weight loss induced by laparoscopic adjustable gastric banding on arterial stiffness, metabolic and inflammatory parameters in patients with morbid obesity. Int J Obes (Lond). 2006; 30:1632-8.

38. Fox AA, Thompson JL, Butterfield GE, Gylfadottir U, Moynihan S, Spiller G. Effects of diet and exercise on common cardiovascular disease risk factors in moderately obese older women. Am J Clin Nutr. 1996; 63:225-33.
39. Kaplan RM, Wilson DK, Hartwell SL, Merino KL, Wallace JP. Prospective evaluation of HDL cholesterol changes after diet and physical conditioning programs for patients with type II diabetes mellitus. Diabetes Care. 1985; 8:343-8.

40. Volek JS, Vanheest JL, Forsythe CE. Diet and exercise for weight loss: a review of current issues. Sports Med. 2005; 35:1-9.

41. Wan Mohamud WN, Musa KI, Md Khir AS, Ismail AS, Ismail IS, Kadir KA, Kamaruddin NA, Yaacob NA, Mustafa N, Ali O, Isa SH, Wan Bebakar WM. Prevalence of overweight and obesity among adult Malaysians: an update. Asia Pac J Clin Nutr. 2011; 20: 35-41. 